LU TP 03-33

hep-ph/0307082

July 2003

\title{
CHIRAL DYNAMICS IN THE MESON SECTOR AT TWO LOOPS ${ }^{\dagger}$
}

\author{
Johan Bijnens \\ Department of Theoretical Physics 2, Lund University, \\ Sölvegatan 14A, S 22362 Lund, Sweden
}

\begin{abstract}
I give a very short introduction to Chiral Perturbation Theory and an overview of the next-to-next-to-leading order three-flavour calculations done. I discuss those relevant for an improvement in the accuracy of the measurement of $V_{u s}$ in more detail. One major conclusion is that all needed $p^{6}$ low energy constants can be obtained from experiment via the scalar form-factor in $K_{\ell 3}$ decays.
\end{abstract}

$\dagger$ Talk presented at CIPANP 2003, Conference on the Intersections of Particle and Nuclear Physics, New York May 19-24, 2003. 


\title{
Chiral Dynamics in the Meson Sector at two Loops
}

\author{
Johan Bijnens \\ Department of Theoretical Physics 2, Lund University, \\ Sölvegatan 14A, S 22362 Lund, Sweden
}

\begin{abstract}
I give a very short introduction to Chiral Perturbation Theory and an overview of the next-to-next-to-leading order three-flavour calculations done. I discuss those relevant for an improvement in the accuracy of the measurement of $V_{u s}$ in more detail. One major conclusion is that all needed $p^{6}$ low energy constants can be obtained from experiment via the scalar form-factor in $K_{\ell 3}$ decays.
\end{abstract}

\section{INTRODUCTION}

The problem of dynamics of mesons at low energies is important. It plays a major role in the precise determination of the elements of the Cabibbo-Kobayashi-Maskawa matrix (CKM) which is a main part of the study of the standard model flavour sector [1]. In this talk I will concentrate on the theory behind the measurement of $V_{u s}$ from $K_{\ell 3}(K \rightarrow \pi \ell v)$ decays and in particular on the recent work of P. Talavera and myself on the two-loop calculation and the determination of the relevant low-energy constants [2, 3].

I review shortly Chiral Perturbation Theory (ChPT) and the relevant two-loop calculations done up to now, followed by a discussion of $K_{\ell 3}$ and the present theory situation. I include a short discussion of the validity of the linear approximation of the form factors normally used in the data analysis. The main relevant results from ChPT [2] can be summarized as follows. The curvatures are important in the analysis but can be predicted using ChPT from the pion electromagnetic form-factor [2] and all order $p^{6}$ parameters needed to determine $V_{u s}$ can be experimentally obtained via the scalar form factor, $f_{0}(t)$, in $K_{\ell 3}$ [2]. The curvature of $f_{0}(t)$ can be predicted as well from knowledge about scalar form factors of the pion [4], albeit only at fairly low precision at present.

\section{CHIRAL PERTURBATION THEORY}

ChPT is an effective field theory valid as an approximation to Quantum Chromodynamics (QCD) at low energies. Its modern form has was introduced by Weinberg, Gasser and Leutwyler [5, 6]. The global chiral symmetry, $S U(3)_{L} \times S U(3)_{R}$, of QCD in the limit of massless quarks is spontaneously broken down to the diagonal subgroup $S U(3)_{V}$ by a nonzero quark condensate, $\langle\bar{q} q\rangle=\left\langle\bar{q}_{L} q_{R}+\bar{q}_{R} q_{L}\right\rangle \neq 0$. The eight broken generators lead to eight Goldstone bosons. These are massless and their interactions vanish at zero momentum. The latter allows the construction of a well defined perturbative expansion in 
terms of momenta, generically referred to as an expansion in $p^{2}$. Quark masses are usually counted as order $p^{2}$ since $p_{\pi}^{2}=m_{\pi}^{2} \sim m_{q}\langle\bar{q} q\rangle / F_{\pi}^{2}$. Inserting an external photon or $W^{ \pm}$-bosons counts as order $p$ since these are included via covariant derivatives. Recent lectures, much more detailed than what is included here are [7]. ChPT being an effective field theory implies that the number of parameters increases order by order. In the purely mesonic strong and semi-leptonic sector there are two parameters at lowest order $\left(p^{2}\right)$, ten at NLO $\left(p^{4}\right)[6]$, and 90 at NNLO $\left(p^{6}\right)$ [8]. The renormalization procedure and the divergences are worked out in general to NNLO [9] and provide a good check on all calculations. One problem shared with other high order loop calculations in comparing different calculations is the use of different renormalization schemes. The calculations that were used to determine all the needed parameters are those of the masses and decay constants [10], $K_{\ell 4}$ [11] and the electromagnetic form factors [12].

\section{$K_{\ell 3}$ : DEFINITIONS, $V_{u s}$ AND FORM FACTOR LINEARITY}

The neutral and charged $K_{\ell 3}$ decays amplitudes, $K^{+, 0}(p) \rightarrow \pi^{0,-}\left(p^{\prime}\right) \ell^{+}\left(p_{\ell}\right) v_{\ell}\left(p_{v}\right)$, are

$$
\begin{aligned}
T^{(+, 0)} & =\frac{G_{F}}{\sqrt{2}} V_{u s}^{\star} \ell^{\mu} F_{\mu}{ }^{(+, 0)}\left(p^{\prime}, p\right), \quad \ell^{\mu}=\bar{u}\left(p_{v}\right) \gamma^{\mu}\left(1-\gamma_{5}\right) v\left(p_{\ell}\right), \\
F_{\mu}^{+, 0}\left(p^{\prime}, p\right) & =(1 / \sqrt{2}, 1)\left[\left(p^{\prime}+p\right)_{\mu} f_{+}^{K^{+} \pi^{0}, K^{0} \pi^{-}}(t)+\left(p-p^{\prime}\right) \mu f_{-}^{K^{+} \pi^{0}, K^{0} \pi^{-}}(t)\right] .(1)
\end{aligned}
$$

Isospin leads to the relations

$$
f_{+}^{K^{0} \pi^{-}}(t)=f_{+}^{K^{+} \pi^{0}}(t)=f_{+}(t) \quad \text { and } \quad f_{-}^{K^{0} \pi^{-}}(t)=f_{-}^{K^{+}} \pi^{0}(t)=f_{-}(t),
$$

The scalar form factor and the usual linear parameterizations are defined as

$$
f_{0}(t)=f_{+}(t)+t /\left(m_{K}^{2}-m_{\pi}^{2}\right) f_{-}(t), \quad f_{+, 0}(t)=f_{+}(0)\left(1+\lambda_{+, 0} t / m_{\pi}^{2}\right) .
$$

To determine $\left|V_{u s}\right|$ we need $f_{+}(0)$ theoretically and experimentally. There are three main theoretical effects. There is a well-known short-distance correction from $G_{\mu}$ to $G_{F}$ calculated by Marciano and Sirlin. The corrections of order $\left(m_{s}-\hat{m}\right)^{2}$ allowed by the Behrends-Sirlin-Ademollo-Gatto theorem are discussed at $p^{6}$ here and in [2, 3]. The sizable isospin breaking found by Leutwyler and Roos [13] is in the process of being evaluated at order $p^{6}$ too. On the experimental side, the old radiative correction calculations used in [13] are updated in [14] where a clean procedure with generalized form-factors is proposed. The experimental data are mostly analyzed using a linear form factor $f_{+}(t)$. The recent precise CPLEAR data [15] allow to test this assumption [2]. Using a linear fit to their data and neglecting systematic errors we get a normalized $f_{+}(0)=1$ and $\lambda_{+}=0.0245 \pm 0.0006$. Allowing for curvature we obtain a sizable curvature, $f_{+}(0)=1.008 \pm 0.009$ and $\lambda_{+}=0.0181 \pm 0.0068$. The fitted curvature is compatible with zero, the central value is precisely at the ChPT prediction given below. In order to obtain $\left|V_{u s}\right|$ with an error of $1 \%$ it is therefore important to include the effect of curvature in the analysis. The central value of $\lambda_{+}$is outside the errors quoted for the linear fit [15]. After the meeting we obtained similar conclusions for the KEK-PS E246 data [16], see v2 of [2]. 


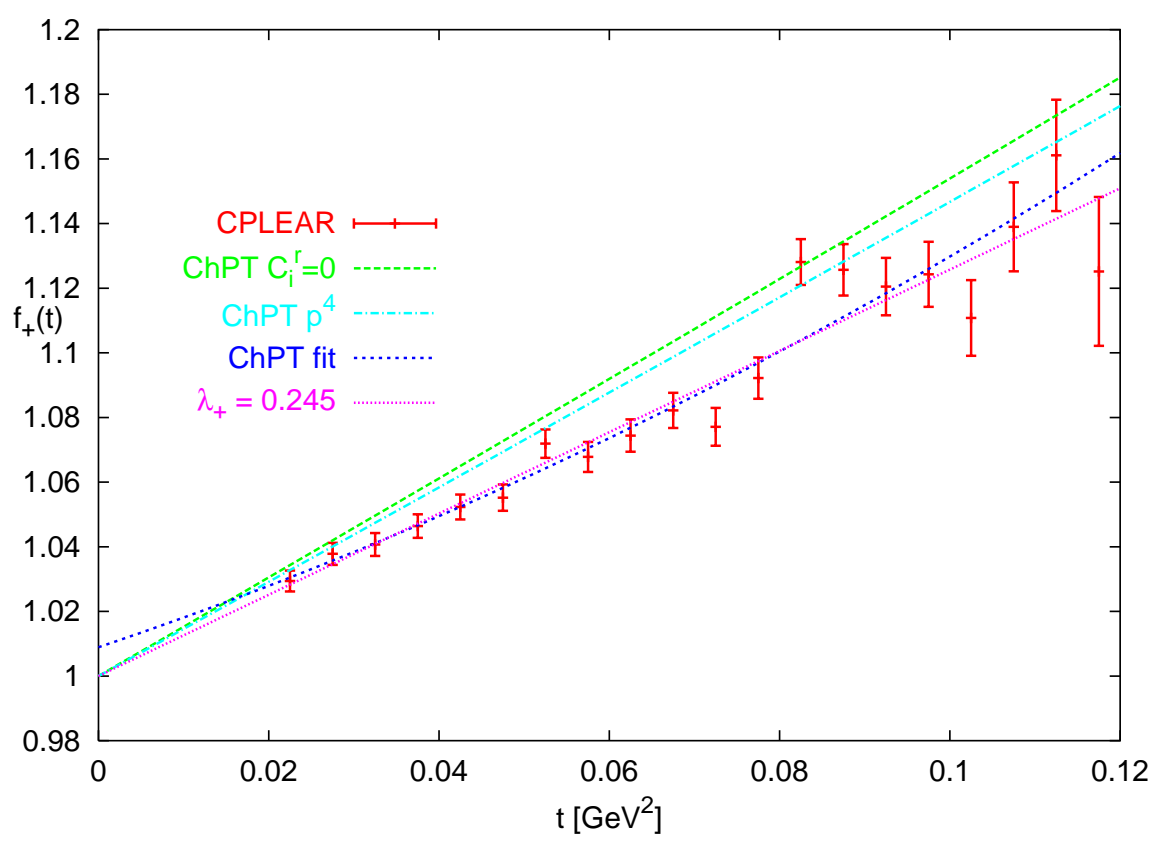

FIGURE 1. ChPT fits to the CPLEAR data showing the effect of the curvature compared to linear fits.

\section{$f_{+}(t)$ AND $f_{0}(t):$ THEORY}

The $f_{+}(t)$ ChPT calculation for is rather cumbersome. I will use our work, [2] but an independent calculation exists [17] and agrees reasonably well, the difference is discussed in [2]. We write the amplitude as

$$
\begin{aligned}
f_{+}(t) & =1+f_{+}^{(4)}(t) / F_{\pi}^{2}+f_{+}^{(6)}(t) / F_{\pi}^{4} \quad \text { with } f_{+}^{(4)}(t)=t L_{9}^{r} / 2+\text { loops } \\
f_{+}^{(6)}(t) & =-8\left(C_{12}^{r}+C_{34}^{r}\right)\left(m_{K}^{2}-m_{\pi}^{2}\right)^{2}+t R_{+1}^{K \pi}+t^{2}\left(-4 C_{88}^{r}+4 C_{90}^{r}\right)+\operatorname{loops}\left(L_{i}^{r}\right)
\end{aligned}
$$

The pion electromagnetic form factor data yield [12] $L_{9}^{r}=0.00593 \pm 0.00043$ and $-4 C_{88}^{r}+4 C_{90}^{r}=0.00022 \pm 0.00002$. With this input we fit the CPLEAR data and obtain

$$
R_{+1}^{K \pi}=-(4.7 \pm 0.5) 10^{-5} \mathrm{GeV}^{2} \quad \text { and } \quad \lambda_{+}=0.0170 \pm 0.0015 .
$$

The first agrees with the VMD estimate [2] $\left.R_{+1}^{K \pi}\right|_{V M D} \approx-410^{-5} \mathrm{GeV}^{2}$. The latter comes from ChPT as

$$
\lambda_{+}=0.0283\left(p^{4}\right)+0.0011\left(\operatorname{loops} p^{6}\right)-0.0124\left(C_{i}^{r}\right)
$$

Our main conclusion follows from rewriting the full $p^{6}$ result for $f_{0}(t)$ as

$$
\begin{aligned}
f_{0}(t)= & 1-\left(8 / F_{\pi}^{4}\right)\left(C_{12}^{r}+C_{34}^{r}\right)\left(m_{K}^{2}-m_{\pi}^{2}\right)^{2}+\left(8 t / F_{\pi}^{4}\right)\left(2 C_{12}^{r}+C_{34}^{r}\right)\left(m_{K}^{2}+m_{\pi}^{2}\right) \\
& +t /\left(m_{K}^{2}-m_{\pi}^{2}\right)\left(F_{K} / F_{\pi}-1\right)-\left(8 t^{2} / F_{\pi}^{4}\right) C_{12}^{r}+\bar{\Delta}(t)+\Delta(0) .
\end{aligned}
$$

$\bar{\Delta}(t)$ and $\Delta(0)$ contain NO $C_{i}^{r}$ and only depend on the $L_{i}^{r}$ at order $p^{6}$ thus ALL needed parameters can be determined experimentally. 
$\Delta(0)=-0.0080 \pm 0.0057[$ loops $] \pm 0.0028\left[L_{i}^{r}\right]$, is known and an expression for $\Delta(t)$ can be found in Ref. [2] The errors are an estimate of higher orders and using fits of the $L_{i}^{r}$ using different assumptions. The $p^{6}$ estimate of [13] corresponds to $-\left(8 / F_{\pi}^{4}\right)\left(C_{12}^{r}+C_{34}^{r}\right)\left(m_{K}^{2}-m_{\pi}^{2}\right)^{2} \approx-0.016 \pm 0.008$.

\section{CONCLUSIONS}

I discussed the $K_{\ell 3}$ form factors in ChPT to order $p^{6}$. The main conclusions are that the curvatures for $f_{+}(t)$ and $f_{0}(t)$ can be predicted from the data on pion electromagnetic [12] and scalar [4] form-factors, the curvature in $f_{+}(t)$ and $f_{0}(t)$ should be taken into account in new precision experiments but from the slope and the curvature we can determine experimentally the needed parameters to calculate $f_{+}(0)$. A precision of better than one percent seems feasible for $\left|V_{u s}\right|$.

\section{ACKNOWLEDGMENTS}

This work has been funded in part by the Swedish Research Council and the European Union RTN network, Contract No. HPRN-CT-2002-00311 (EURIDICE)

\section{REFERENCES}

1. M. Battaglia et al., hep-ph/0304132

2. J. Bijnens and P. Talavera, hep-ph/0303103 to be published in Nucl. Phys. B.

3. J. Bijnens, Talk given at 38th Rencontres de Moriond on QCD and High-Energy Hadronic Interactions, Les Arcs, Savoie, France, 22-29 Mar 2003, hep-ph/0304284

4. J. Bijnens and P. Dhonte, hep-ph/0307044

5. S. Weinberg, Physica A 96 (1979) 327; J. Gasser and H. Leutwyler, Annals Phys. 158 (1984) 142,

6. J. Gasser and H. Leutwyler, Nucl. Phys. B 250 (1985) 465.

7. A. Pich, A., hep-ph/9806303, G. Ecker, hep-ph/0011026, S. Scherer, hep-ph/0210398

8. J. Bijnens, G. Colangelo and G. Ecker, JHEP 9902 (1999) 020 [hep-ph/9902437|;

9. J. Bijnens, G. Colangelo and G. Ecker, Annals Phys. 280 (2000) 100 |hep-ph/9907333|.

10. G. Amorós, J. Bijnens and P. Talavera, Nucl. Phys. B 568 (2000) 319 |hep-ph/9907264|, Nucl. Phys. B 602 (2001) 87 |hep-ph/0101127|.

11. G. Amorós, J. Bijnens and P. Talavera, Phys. Lett. B 480 (2000) 71 [hep-ph/9912398]; Nucl. Phys. B 585 (2000) 293 [Erratum-ibid. B 598 (2001) 665] |hep-ph/0003258|.

12. J. Bijnens and P. Talavera, JHEP 0203 (2002) 046 |hep-ph/0203049|.

13. H. Leutwyler and M. Roos, Z. Phys. C 25 (1984) 91.

14. V. Cirigliano et al., Eur. Phys. J. C 23 (2002) 121 |hep-ph/0110153|.

15. A. Apostolakis et al. [CPLEAR Collaboration], Phys. Lett. B 473 (2000) 186.

16. A. S. Levchenko et al. [KEK-PS E246 Collaboration], Phys. Atom. Nucl. 65 (2002) 2232 [Yad. Fiz. 65 (2002) 2294] |hep-ex/0111048|.

17. P. Post and K. Schilcher, Eur. Phys. J. C 25 (2002) 427 |hep-ph/0112352|. 